\title{
Extensions, Simplifications, and Tests of Synchronic Modal Equivalencing (SME)
}

\author{
Ganesh N. Ramaswamy $\oint^{\dagger}$ Christophe Evrard ${ }^{\dagger} \quad$ George C. Verghese ${ }^{\ddagger *}$ \\ OLlivier Fillâtre ${ }^{\dagger}$ Bernard C. LESIEUTRE ${ }^{\ddagger *}$ \\ ${ }^{\ddagger}$ Massachusetts Institute of Technology, Laboratory for Electromagnetic and Electronic Systems, Cambridge, MA 02139 \\ ${ }^{\dagger}$ Electricité de France, Direction des Etudes et Recherches, 1, ave. du Général de Gaulle, 92141 Clamart, France
}

\begin{abstract}
Synchronic Modal Equivalencing (SME) is aimed at structure-preserving dynamic equivalencing of large power system models. SME is motivated by the slow-coherency method for system partitioning and aggregation, but its formulation and associated computational algorithms are more general in some important respects. Ideal synchrony of a group of generators requires the motion of each generator in the group to be a linear combination of the motions of a fixed set of basis generators in the group, when some subset of the system modes (not necessarily the slowest) is excited. SME keeps intact the dynamic models of all the generators in a synchronic study group and of all the basis generators external to this group. The model of each remaining generator is replaced by a simple nondynamic linear circuit containing a dependent current source driven by the motions of the basis generators. The remainder of the network can be left unmodified.
\end{abstract}

Previous numerical tests of SME have been limited to relatively small and simple models. This paper makes the transition to a detailed, intermediate-sized model, while also introducing certain extensions and simplifications that open the door to experiments with much larger models. A recommended SME procedure is specified in the paper, and illustrated with tests performed using the simulation package EUROSTAG and a model loosely based on the France-Spain power system. The unreduced model has 23 generators, 83 buses, and 476 state variables; the model is reduced in a structure-preserving way to 10 generators and 255 state variables, with very good results.

Key Words - Aggregation, Decomposition, Dynamic Equivalents, Modal Equivalents, Partitioning, SlowCoherency, Synchrony.

96 SM 538-9 PWRS A paper recommended and approved by the IEEE Power System Engineering Committee of the IEEE Power Engineering Society for presentation at the 1996 IEEE/PES Summer Meeting. July 28 - August 1, 1996, in Denver. Colorado. Manuscript submitted December 29, 1995; made available for printing June 27 , 1996

\section{Introduction}

The process of dynamic equivalencing, i.e. the systematic construction of reduced-order or aggregated dynamic models that preserve behavior of interest, is of considerable importance in power systems. The state of the art for serious application in the transient stability context is represented in the package DYNRED [1], which is built on the classical coherency-based equivalencing approach in [2]. DYNRED also incorporates more recent ideas from slow-coherency/weak-coupling theory, [3], [4], [5], to identify coherent groups of generators in a large model. The slow-coherency approach actually yields direct possibilities for equivalencing, although these have been explicitly addressed only for the case of low-order generator models that capture just the swing dynamics, see [5], [6]. Yet other approaches to dynamic equivalencing have been proposed in the literature, see for instance [7].

A new framework for dynamic equivalencing, termed Synchronic Modal Equivalencing (SME), was introduced in [8], [9]. The objectives of the current paper are to:

- summarize certain extensions and simplifications of SME that have been obtained in the thesis [10] (partially reported in [11]) and in more recent work;

- lay out the recommended SME procedure that emerges from our studies so far; and

- describe the results of tests on a model that is larger and more detailed than those used in our earlier work.

SME is motivated by slow-coherency theory, but its formulation and associated computational algorithms are more general in some important respects. Ideal slow-coherency of a group of generators requires that they exhibit identical motions when only the slow behavior of the system is excited. On the other hand, ideal synchrony of a group of generators requires the motion of each generator in the group to be a linear combination of the motions of a fixed set of basis generators in the group, when some subset of the system modes

\footnotetext{
*Address for correspondence: Room 10-050, Laboratory for Electromagnetic and Electronic Systems, Massachusetts Institute of Technology, Cambridge, MA 02139

$\S$ Previously at MIT; now at IBM Thomas J. Watson Research Center, Route 134, Yorktown Heights, NY 10598
} 
(not necessarily the slowest) is excited; we refer to this subset of modes as the chord in which synchrony is displayed. This extended definition of synchrony was introduced in [10], [11]; our earlier definition of synchrony in [8], [9], [12] actually corresponds to the more restrictive case of one-dimensional synchrony, where there is only one generator in the basis for each synchronic group. The algorithms developed for SME enable chord selection, recognition of synchronic groups, basis selection, as well as other computations required to construct the SME equivalent.

Section II of this paper outlines the steps that comprise the recommended SME procedure. Interspersed remarks draw attention to extensions and simplifications obtained since [8], [9]. Section III of the paper describes the results of numerical experiments with an intermediate-sized model that is roughly based on the France-Spain power system. By "intermediatesized" we mean that the model is large enough to display nontrivial synchronic groups, but not so large that typical large-system methods such as sparse matrix computations and reduced-order eigenanalysis are mandated. This restriction to intermediate-sized models seems entirely appropriate at this intermediate stage of development of the SME framework.

\section{Recommended SME Procedure}

\section{A. Overview}

The SME procedure for constructing a dynamic equivalent occurs in three stages. The first stage (described in Steps 1,2,3 below) involves preparation of the various dynamic models that are necessary or useful for the process. The second stage (in Steps 4,5) is devoted to analysis of the modal structure of a simple, low-order, linearized, electromechanical model of the system, such as a linearized, undamped, swing-equation model. This analysis leads to the selection of a suitable chord, and to the decomposition of the model into one-dimensional synchronic groups, each associated with a distinct basis generator. One of these synchronic groups (or possibly the union of several synchronic groups) is selected as a study group.

In the third stage (Steps 6,7,8), we return to the original, large, unreduced, nonlinear model of the system, to impose on it the structural decomposition suggested by the preceding analysis of the simplified model. The models of all the generators of the study group and of all the basis generators external to the study group are retained in full nonlinear detail. Each of the remaining "less-relevant" generator models is replaced by a simple nondynamic linear circuit that contains a dependent current source driven by the motions of the basis generators. Impedance-load buses that interconnect only the replaced generators can be eliminated, with their effects being accounted for by including the bus voltages of the replaced generators as additional driving variables for the dependent current sources. The remainder of the network is left unmodified.

Figure 1 is a schematic representation of the structurepreserving reduction that occurs in going from the original model to the final SME equivalent. The precise form of the dependent-source circuit that replaces the less-relevant generators will be developed later (see Fig. 2). Note that in
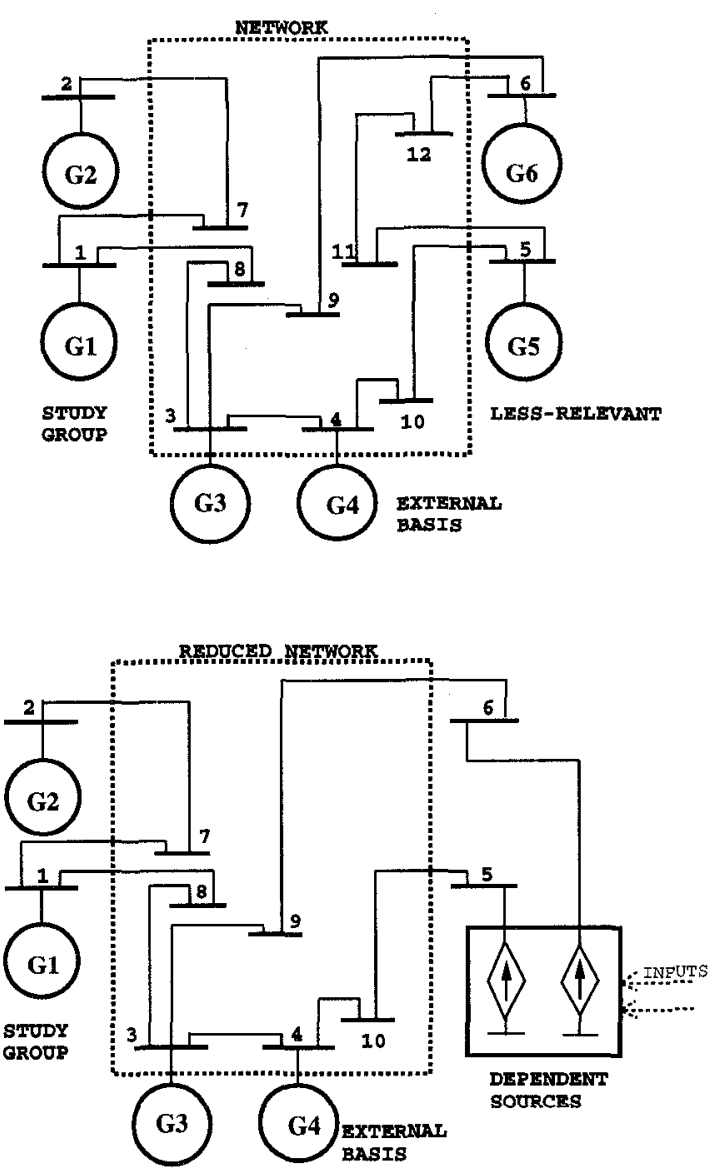

Figure 1: Structure-Preserving Reduction. The top figure is a schematic representation of the large, original, unreduced model. The bottom figure shows the form of the reduced SME equivalent.

practical situations the less-relevant generators are likely to heavily outnumber the retained generators.

\section{B. Rationale}

The rationale for the preceding route to a dynamic equivalent lies in a succession of claims. Both theory and practice show that each of these claims can be justified to some degree, and that each has its limitations. Briefly, the claims are as follows:

- The structure of system-wide dynamics in the power system is substantially reflected in the electromechanical modes of a linearized model of the system. These electromechanical mode frequencies and mode shapes are in turn well-represented in a simple linearized undamped swing-equation model.

- Slow-coherency theory shows that in an ideal case the undamped linearized swing-equation model displays wellseparated slow and fast modes, with groups of generators moving coherently in the slow modes, and with the fast modes being essentially confined to one or another 
of these slow-coherent groups. In other words, the slow modes constitute the inter-group dynamics, while the fast modes constitute the intra-group dynamics.

- Thus, to reproduce all the motions of a selected slowcoherent study group in this idealized case, one needs only to retain all the generators in the study group, as well as a single representative generator from each of the other slow-coherent groups.

- Regions distant from a disturbance or fault will be perturbed sufficiently slightly that calculations with linearized models can be relevant.

The above claims have all been presented and argued in prior literature. The following claims are more particular to SME, as justifications for extending the notion of slow-coherency to synchrony:

- The slow-coherency theory is an asymptotic theory that describes what happens in the idealized limit of vanishingly small couplings between separate groups of machines. In practice, it is often not easy to make a clear separation into slow and fast behavior; the modal frequencies tend to run together.

- More seriously, even when inter-group dynamics are present in practice, they can occur in a chord that is not necessarily the slowest. The thesis [10] presents several examples that show the importance of the flexibility to pick chords other than the slowest, in non-ideal situations. A sequential algorithm aimed at picking a chord that corresponds to well-defined inter-group and intra-group dynamics is presented in [12]. A criterion for picking the size of the chord is noted later in this paper.

- The intra-group dynamics seen in practice will more likely involve linear relations among the motions of the generators in a group, rather than equality of motions.

Given the complexity of the context in which dynamic equivalencing is carried out, the real validation of any approach to it has to lie in the quality of the results one obtains in practice, and the ease with which these results are obtained. Our tests with intermediate-sized models, as reported in this paper, show SME to be promising on both counts. Tests on the much larger models that are ultimately of interest to industry will be carried out as the next stage of this research effort. This phase will undoubtedly bring its own set of challenges, but our results so far permit some optimism.

\section{SME Procedure}

Step 1: Form a detailed nonlinear model and determine its nominal operating point

The original detailed model of the power system typically (and most conveniently) is assembled in the form of a system of differential-algebraic equations (DAEs) whose structure reflects that of the underlying system:

$$
\begin{cases}\dot{X}(t) & =f(X(t), W(t)) \\ 0 & =g(X(t), W(t))\end{cases}
$$

where $X(t)$ is the vector of differential (state) variables at time $t$, and $W(t)$ is the vector of algebraic variables. The nominal equilibrium or operating point corresponds to constant solutions $X(t)=\bar{X}, W(t)=\bar{W}$, and would be found by a standard steady-state analysis.

\section{Step 2: Linearize about the operating point}

The linearized model describes small perturbations of the variables from their equilibrium values: $x(t)=X(t)-\bar{X}$ and $w(t)=W(t)-\bar{W}$. Most of the work of linearization can in fact be done analytically, since models for most power system components are analytically specified.

If we use the linearized algebraic equations to eliminate the perturbations in all variables except the bus voltages, a $\mathrm{DAE}$ model of the following form can be obtained:

$$
\left\{\begin{array}{l}
\dot{x}(t)=A_{d} x(t)+B_{d} u(t) \\
0=C_{d} x(t)+\left(D_{d}-Y\right) u(t)
\end{array}\right.
$$

where: $u(t)$ denotes the perturbation of the vector $U(t)$ of bus voltages from its nominal value $\bar{U}$; the subscript $d$ denotes block-diagonal matrices whose individual blocks correspond to the various subsystems (generators and loads) connected to the buses; $C_{d} x(t)+D_{d} u(t)$ denotes the vector of current perturbations injected into the network; and $Y$ denotes the admittance matrix of the transmission network.

The DAE model in (2) would be used for all subsequent computations that require an unreduced linearized model. However, for intermediate-sized systems such as the one tackled in this paper, the computational burden of reducing (2) all the way to state-space form is insignificant, and the convenience of having available a state-space model during this phase in the development and testing of SME is substantial. The state-space model is obtained by eliminating $u(t)$ from (2). Using the notation

$$
J=D_{d}-Y
$$

we get a linear, time-invariant model in standard state-space form:

$$
\dot{x}(t)=\left[A_{d}-B_{d} J^{-1} C_{d}\right] x(t)=A x(t)
$$

In most of what follows, the time argument $t$ will be dropped for notational simplicity.

\section{Step 3: Obtain a simple linearized model of the un-} damped swing dynamics

For this step, our earlier work, [8], [9], used a linearized undamped swing-equation model that was constructed directly. However, it can be more convenient to extract a model with essentially the same features as this swing-equation representation from the DAE model (2) or the state-space model (4). If (4) is the starting point, then among the state variables in $x$ one almost invariably has the generator (angular) position. and velocity perturbation vectors $\delta$ and $\omega$ respectively. Some of the rows of (4) therefore constitute an equation of the form

$$
\dot{\omega}(=\ddot{\delta})=\mathcal{A} \delta+\cdots
$$

where "..." stands for the dependence of $\dot{\omega}$ on components of $x$ other than $\delta$. Ignoring this dependence on other variables, we will typically have in (5) a model of the desired 
form; we shall refer to the resulting model as the core model. Its order equals the number of generators in the system. The core model can also be obtained directly from (2), which requires less computation than first obtaining (4). A possible advantage of the core model over the standard swing-equation model is that its features may be more easily correlated with the detailed model (2). However, the linearized, undamped swing-equation model can be used instead of the core model in all that follows.

The matrix $\mathcal{A}$ in (5) is diagonally similar to a symmetric, negative semi-definite matrix. Thus $\mathcal{A}$ possesses one eigenvalue at 0 , with all other eigenvalues real and negative; we denote these eigenvalues, in decreasing order, by $\lambda_{0}(=0), \lambda_{1}, \lambda_{2}, \ldots$. The natural frequencies of the core model are thus the (purely imaginary) square roots of the $\lambda_{i}$, and constitute good estimates of the swing-mode frequencies. The associated mode shapes for $\delta$ (and also for $\omega$ ) are indicated by the corresponding eigenvectors of $\mathcal{A}$, which are all real.

Step 4: For the simple model, select a good chord, find basis generators for one-dimensional synchrony, and form synchronic groups

The required computations for this stage are described in [12] and [10]. Note that basis generators for one-dimensional synchronic groups were referred to as reference generators in [12]; our present terminology seems preferable, in view of the extended (multi-dimensional) notion of synchrony that we are using now.

Recognizing synchrony: Synchrony in a given chord of (5) can be recognized by examining the rows of the chordal matrix, a matrix whose columns are the eigenvectors of $\mathcal{A}$ that are associated with the chord. Note that each row of the chordal matrix (or chordal row) corresponds to a particular generator. A generator exhibits exact one-dimensional synchrony with a basis generator if its corresponding chordal row is a (positive or negative) multiple of the chordal row of the basis generator. Approximate synchrony involves approximate alignment of these chordal rows, and measures of synchrony aim to quantify the degree of alignment. The degree of synchrony between two generators is defined in [12] as the cosine of the angle between the corresponding chordal rows. The synchronic distance of a given generator from a basis generator is defined in [10] as the distance between its chordal row and the projection of this row on the chordal row of the basis generator. For both these measures, it can be advantageous to work with weighted versions of the chordal rows, as noted in [10], [12].

Finding basis generators: To pick the basis generators for a given chord, the thesis [10] presents an algorithm that is considerably simpler than the fuzzy clustering procedure originally prescribed in [12]. In this simplified algorithm, the first basis generator picked is the one with the largest total participation, [13], in the chosen chord, where this total is measured in some convenient way. The second basis generator is picked as the one that has the largest synchronic distance to the first basis generator. We continue in this fashion until the desired number of basis machines has been obtained, each time picking the next basis generator so as to maximize its minimum synchronic distance from existing basis generators.

Forming synchronic groups: Given a set of basis generators, the definition of synchronic groups is carried out by assigning each non-basis generator to the basis generator with which it has the highest-magnitude degree of synchrony.

Selecting a good chord: Chord selection proceeds sequentially. We start with two modes of the model (5), perhaps the slowest or most extensive, then look for three synchronic groups in this chord, using the procedure described in the preceding subsections. Three approximately inter-group modes can be associated with this grouping; two of these modes will be good approximations to the chord we began with, while the third will be a good approximation to an additional mode of (5). This additional mode is now added to our chord, and the procedure is continued, searching for four groups in the three-mode chord. The process terminates when we obtain a chord with the desired number of modes, which is also the desired number of synchronic groups. It is shown in [10] that this procedure is consistent with obtaining well-defined intergroup and intra-group behavior in the final chord.

For dynamic equivalencing of systems with under around 500 generators, which covers a great number of situations of practical interest (especially as one moves towards using such equivalents to enable near-real-time applications), a full eigenanalysis of the core model is quite tractable, so chord selection can be done with a full picture of the modal structure of the system. However, our sequential chord selection procedure is also amenable to sequential computation of just the selected modes (each one of which comes with an automatically generated initial guess), if the size of the core model precludes full eigenanalysis.

Choosing the number of groups: We are still left with the problem of determining the number of modes to use in a chord, or equivalently, the number of synchronic groups to end up with. A desirable choice would be one for which the largest intra-group synchronic distance is small, relative to the smallest inter-group synchronic distance, because this would correspond to having distinct, well-defined synchronic groups. Other considerations include the extent of reduction one seeks, practical constraints on the choice of a study group (see Step 5 below), and so on.

\section{Step 5: Select a study group}

The choice of which synchronic group (or groups, taken together) should constitute the study group depends on the particular events that one wishes to investigate. The study group should clearly include any generators that are to be disturbed. For disturbances on load buses or transmission lines that are not tightly associated with the study area alone, the proper choice of study group may not be so evident; further research and experience are needed to refine the selection of the study group for such situations. The guiding principle, however, is that the intra-group modes of external (i.e. nonstudy) synchronic groups should not be significantly excited by the disturbances of interest. This in turn implies that the study group should comprise one or more entire synchronic groups, not portions of such groups. Thus a study group should not be arbitrarily defined; the decomposition into synchronic groups shows where the allowed "boundaries" of a 
study group may fall. (Although we talk of boundaries, the fact that only generators and not load buses or transmission lines are assigned to synchronic groups means that we are not really talking of geographic partitions.)

The basis generators inherited from the earlier decomposition into one-dimensional synchronic groups (see Step 4) may be expected to work well as basis machines for whichever group, study or external, they fall into. We shall therefore retain them for what follows. However, it is possible to revisit the selection of basis generators for the study and external areas, to optimize the performance in the steps below; some initial explorations are found in the thesis [14].

Step 6: Impose synchrony-derived structure on the original, unreduced model

We now return to the detailed linearized model (2) to impose on it the structure exposed by analysis of the simplified model (5). Denote by $x_{z}$ the variables in $x$ that correspond to the less-relevant (i.e. non-basis, external) generators; call these the less-relevant variables. Let $x_{r}$ denote the remaining (relevant) variables, which include the variables in $x$ that correspond to the study-group generators and to the external basis generators. After reordering (2) to expose the relevant and less-relevant parts, the linearized DAE model becomes

$$
\left(\begin{array}{c}
\dot{x}_{r} \\
0 \\
0 \\
\dot{x}_{z}
\end{array}\right)=\left(\begin{array}{cccc}
A_{d r} & B_{d r} & 0 & 0 \\
C_{d r} & J_{r r} & J_{r z} & 0 \\
0 & J_{z r} & J_{z z} & C_{d z} \\
0 & 0 & B_{d z} & A_{d z}
\end{array}\right)\left(\begin{array}{c}
x_{r} \\
u_{r} \\
u_{z} \\
x_{z}
\end{array}\right)
$$

where $u_{r}$ and $u_{z}$ are the vectors of voltage perturbations at the buses of the relevant and remaining buses respectively. The vector $x_{r}$ can itself be separated into variables $x_{b}$ associated with the basis generators, and the remaining relevant variables $x_{\rho}$. After some reordering of variables, if necessary, we can write

$$
x_{r}=\left(\begin{array}{l}
x_{b} \\
x_{\rho}
\end{array}\right)
$$

For our work, we shall take $x_{b}$ to contain just the $\delta$ and $\omega$ components of the basis generators. The numerical experiments reported in the next section involve two different choices of basis machines: for a full basis, we use the external basis generators and the study-group basis generator; for an external basis, we only use the external basis generators.

\section{Step 7: Obtain the form of the SME equivalent}

Suppose now that a relation of the form

$$
x_{z}=K_{b} x_{b}=\left(\begin{array}{ll}
K_{b} & 0
\end{array}\right)\left(\begin{array}{l}
x_{b} \\
x_{\rho}
\end{array}\right)=K x_{r}
$$

happens to hold (at least approximately) for some matrix $K_{b}$, for disturbances in the study group. The reasons for anticipating such a relation will be outlined in Step 8, where we show how $K_{b}$ may be computed. Given (8), we can condense (6) to the form

$$
\left(\begin{array}{c}
\dot{x}_{r} \\
0 \\
0
\end{array}\right)=\left(\begin{array}{ccc}
A_{d r} & B_{d r} & 0 \\
C_{d r} & J_{r r} & J_{r z} \\
C_{d z} K & J_{z r} & J_{z z}
\end{array}\right)\left(\begin{array}{c}
x_{r} \\
u_{r} \\
u_{z}
\end{array}\right)
$$

This is the form of the SME equivalent. The route to this equivalent is much more direct and fruitful than our earlier

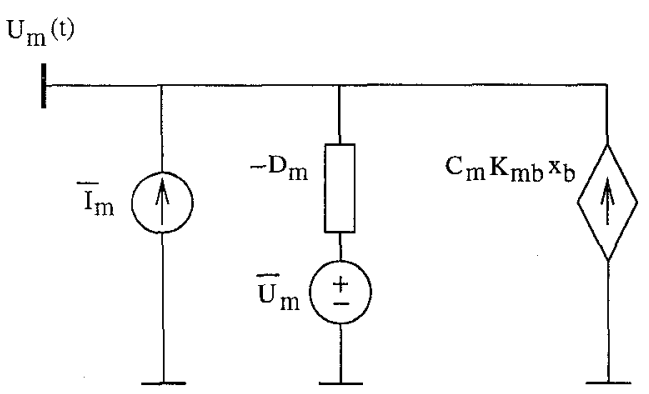

Figure 2: Replacement for the $m$ th Less-Relevant Generator. Here $\bar{I}_{m}$ denotes the nominal output current of the generator; $\bar{U}_{m}$ is the nominal terminal bus voltage; the term $-D_{m}$ is the small-signal admittance of the generator; and the nondynamic dependent current source $C_{m} K_{m b} x_{b}$ represents the $m$ th block row of $C_{d z} K_{b} x_{b}$, where $C_{m}$ is the $m$ th diagonal block of $C_{z d}, K_{m b}$ is the $m$ th block row of $K_{b}$, and $x_{b}$ denotes the $\delta$ and $\omega$ components of the basis generators.

approach in [9], which was motivated by ideas of Selective Modal Analysis, [13].

What (9) describes is the original linearized model (6), except that the dynamic contributions (via the term $C_{d z} x_{z}$ ) of the less-relevant generators to the remainder of the system have been replaced by a vector of current injections $C_{d z} K_{b} x_{b}$ at the buses of these generators, i.e. a set of dependent currents driven by the motions of the basis generators. All that is left of the less-relevant generators is their nondynamic part, namely the small-signal admittances $-D_{d z}$ buried in the factor $J_{z z}=D_{d z}-Y_{z z}$ that occurs in (9).

Examination of $Y_{z z}$ can also expose the presence of impedance-load buses (e.g. buses 11 and 12 in the schematic of Fig. 1) that are only connected to each other and to the buses of the less-relevant generators. These load buses can be eliminated by a standard Ward-type reduction, and exactly compensated for by a modification of the dependent current source at the bus of each less-relevant generator, adding terms that depend linearly on the voltages at the buses of all the less-relevant generators. The computational benefits of doing this will depend on the particular system being studied.

We can now translate the above reduction of the linearized model to a reduction of the original nonlinear model (1). Again, only the less-relevant generators are affected. The replacement for the $m$ th less-relevant generator is the configuration shown in Fig. 2, obtained simply by offsetting the results for the linearized case with the appropriate nominal operating generator current $\bar{I}_{m}$ and terminal voltage $\bar{U}_{m}$.

\section{Step 8: Compute the modal correction $K_{b}$}

The reasons for anticipating a relation of the form (8) are as follows:

- For disturbances in the study group, our careful synchronic grouping should ensure that only the inter-group electromechanical modes of the system (2), (4), (6) are significantly excited outside the study group. Consequently, $x_{b}$ and $x_{z}$ will display mainly the inter-group 
modes, except that any variables of the study-group basis generator(s) in $x_{b}$ will also display the intra-group modes of the study group.

- Given synchrony in the inter-group modes, we expect that at least the $\delta$ and $\omega$ portions of $x_{z}$ will be approximately linear combinations of the basis variables when only the inter-group modes are excited.

The above reasoning suggests that it is sufficient to try and satisfy (8) for the case where just the inter-group modes are excited. Also, we can ignore those components of $x_{z}$ that do not contribute to the product $C_{d z} x_{z}$; this consideration typically leaves us with only the $\delta, \omega$ and rotor flux components of the less-relevant machines to worry about. Under these conditions, many strategies are possible. We shall shortly outline just four; some others are considered in [10] and [14], and still more may be imagined.

Proceeding directly, we first find the inter-group modes of $(2),(4),(6)$. These modes were labeled as inter-group modes only in the small, simplified model (5), so we now have the challenge of recognizing them among the (very many more) modes of the large, unreduced linearized model. For this, we have to begin by actually computing these modes for this unreduced model. With intermediate-sized models, the mode computation is routine. With very large models, computing the electromechanical modes can be a difficult task, but effective methods have been developed, see references $\{2\},\{10\},\{16\},\{17\}$ in [9] for example.

The matching of the oscillatory inter-group modes from (5) with modes of the large model can be carried out quite reliably using mode frequencies, mode shapes, and participation factors (taking advantage of the fact that the participation in electromechanical modes is almost entirely contributed by generator angles, velocities, and rotor fluxes). Recall that each eigenvalue $\lambda_{i}$ of $\mathcal{A}$ in (5) corresponds to a pair of eigenvalues at approximately $\pm j \sqrt{-\lambda_{i}}$ for $A$ in (4). On occasion, there may be more than one candidate for a match, and some further exploration may be needed. The eigenvalue $\lambda_{0}=0$ of $\mathcal{A}$ maps to an eigenvalue at 0 for the large model, and another small negative real eigenvalue, which may be termed the partner of the eigenvalue at 0 . However, locating the correct partner eigenvalue can be tricky, so we have dropped this eigenvalue in all our computations with inter-group modes: when we talk of inter-group modes of the unreduced model in what follows, we are referring to the solitary mode at 0 , along with the oscillatory mode pairs that correspond to $\pm j \sqrt{-\lambda_{i}}$ for those $\lambda_{i}$ represented in the chord of interest.

Suppose now that the (chordal) matrix of inter-group eigenvectors for the unreduced model is partitioned conformably with the ordering we have been assuming for the components $x_{b}, x_{p}$, and $x_{z}$ of $x$ :

$$
V=\left(\begin{array}{l}
V_{b} \\
V_{\rho} \\
V_{z}
\end{array}\right)
$$

Then satisfying (8) for the case where just the inter-group modes are excited corresponds to finding a $K_{b}$ that satisfies

$$
V_{z}=K_{b} V_{b}
$$

This equation motivates our referring to the computation of $K_{b}$ as the modal correction step of our SME procedure. There are potential advantages to working with a modified version of (11) in which each row is pre-weighted before solving for $K_{b}$, see [11], [10], but for this paper we stay with the unweighted version.

If we use a full basis, as defined at the end of Step 6 , then $V_{b}$ in (11) has one more row than column, corresponding to an underconstrained set of equations, with an infinite number of solutions. The formula

$$
K_{b}=V_{z} V_{b}^{\dagger}
$$

where $V_{b}^{\dagger}$ denotes the pseudo-inverse of $V_{b}$, gives the solution $K_{b}$ that has the least sum of squared entries, and we refer to this as "the" full-basis/full-mode solution. Note, however, that other solutions are possible, and further research may show one of these alternatives to even be preferable.

An external basis (see Step 6) may be expected to perform better for our purposes, because the study-group basis generator displays the intra-study-group modes in addition to the inter-group modes of interest to us. (The more careful reasoning in [10], [11] shows that this is especially true when the study-group basis generator is directly disturbed.) In this case, $V_{b}$ in (11) has one fewer row than column, corresponding to an overconstrained set of equations, which typically will not have a solution. A possible choice of $K_{b}$ in this case is the least square error solution, still given by the formula in (12). We shall refer to $K_{b}$ computed this way as "the" externalbasis/full-mode solution. Once again, other solutions are possible, and may end up being preferable.

Our experience so far shows that highly satisfactory SME equivalents can in fact be obtained by satisfying (8) for just the mode at 0 , whose eigenvector is immediately available for even the largest power system models; this eigenvector has an entry of 1 for each generator angle, and all its other entries are 0 . If this experience holds up with tests on much larger models than those we have tackled so far, it will imply a great simplification of this step of the SME procedure. Again, the formula in (12) can be used for what is now an underconstrained case. If we use an external basis, we refer to the resulting $K_{b}$ in this case as "the" external-basis/zeromode solution.

Finally, the simplest choice is to take $K_{b}=0$, i.e. ignore the dependent current source in Fig. 2. We refer to this as the no-correction solution. Even this crude choice turns out to perform quite well, as already demonstrated in [9], reflecting how much the preceding steps of the SME procedure are contributing to the equivalent.

\section{Illustrations On A Test Example}

We present in this section an illustration of the SME procedure, and of the results it produces. The model we use is roughly based on the France-Spain power system; the network has 83 buses, and the 23 generators are represented in some detail, with a total of 476 state variables. Our earlier timedomain studies, [12], were limited to a swing-equation model with 93 state variables. 


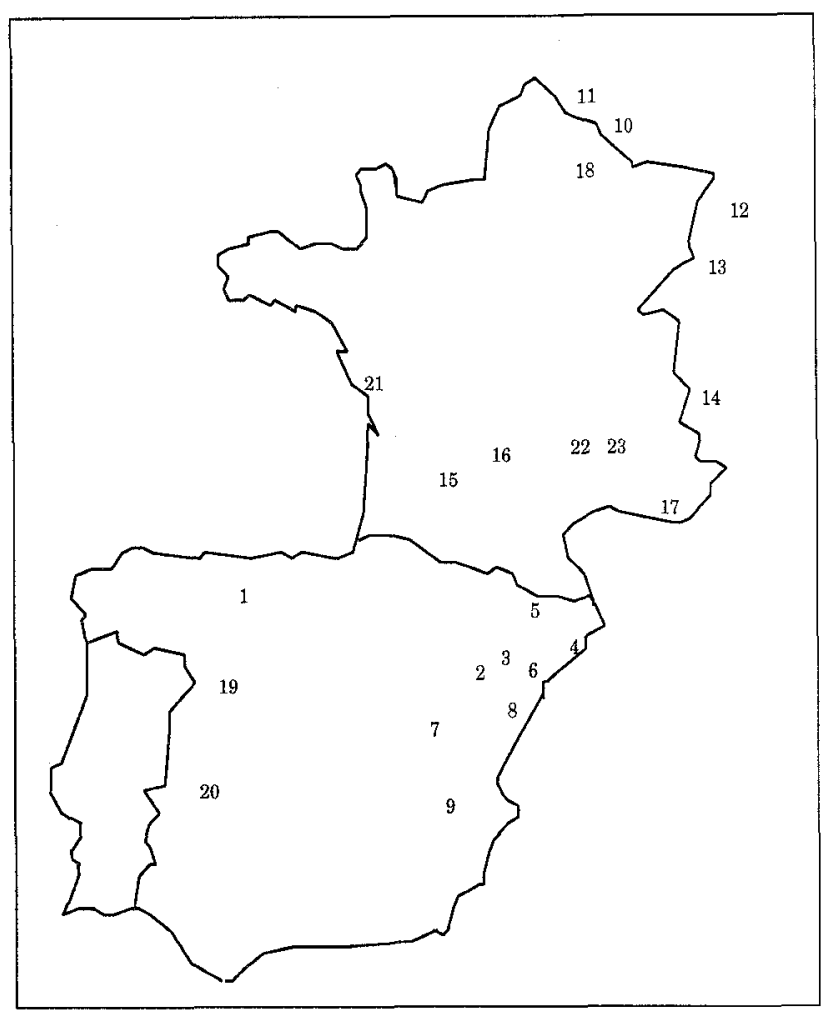

Figure 3: Relative Geographical Locations of the Generating Units in the Test Model. With a chord of five modes, the corresponding one-dimensional synchronic groups were determined to be $\{\mathbf{2 1}, 15, \mathbf{1 6}, 17,22,23\}$ (chosen as the study area), $\{\mathbf{1 3}, 10,11,12\},\{\mathbf{1 4}\},\{\mathbf{1 8}\}$ and $\{\mathbf{1 9}(1$ to 9$)$ $20\}$, with the basis generator shown boldfaced.

\section{A. Procedure}

The detailed unreduced model was implemented in the software package EUROSTAG, [15], [16], and its operating point and linearization at this operating point were determined (Steps 1 and 2). All computations with the linearized model were carried out in MATLAB, [17]. The square matrix $A$ of (4) has dimension 476. The core model extracted from (4) has a matrix $\mathcal{A}$ of dimension 23 (Step 3).

The sequential chord selection process (Step 4) yields modes in the order $\lambda_{0}, \lambda_{1}, \lambda_{6}, \lambda_{4}, \lambda_{3}, \lambda_{7}, \ldots$, which is significantly different from the ordering in ascending magnitude that slow-coherency would end up with. Based on the criterion described at the end of Step 4, and given the desire to have a study group that includes most of the generators in France, the best number of groups for our decomposition turns out to be five. These groups, computed from the chord comprising the first five modes listed above, are indicated in Fig. 3. The group with the machines $\{\mathbf{2 1}, 15,16,17,22$, $23\}$ was then selected (Step 5) to be the study area (the basis generator appears in boldface). We thus have 6 generators in the study area and 4 external basis generators (with 255 associated state variables altogether, which become the state variables of the equivalenced system), and 13 machines that will eventually be reduced. The detailed model can be struc-

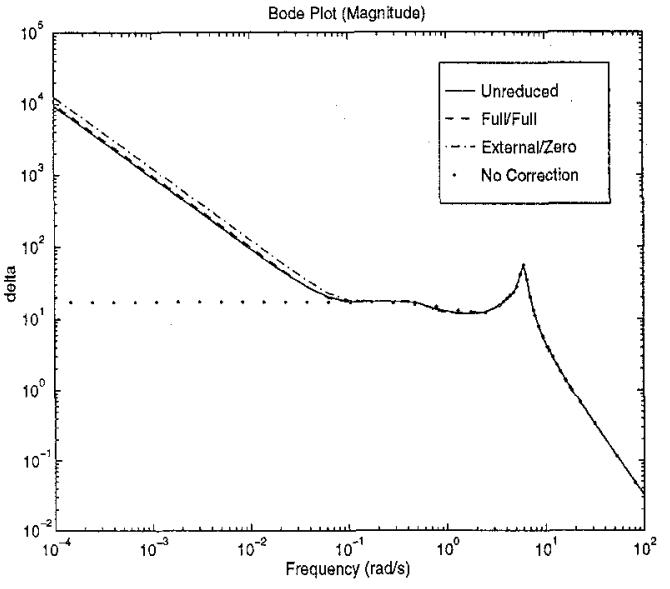

Figure 4: Bode Magnitude Plot for Generator 17. The input is the mechanical power perturbation at generator 17 and the output is the perturbation in the absolute angle of the same machine.

tured accordingly (Step 6).

The form of the SME equivalent can now be obtained (Step 7). The implementation of the dependent current sources for the replaced generators is completely straightforward in EUROSTAG. Finally, we can compute the modal correction term $K_{b}$ in at least the four ways mentioned earlier (Step 8). The results of numerical experiments with the resulting SME equivalents are presented in the next subsection.

\section{B. Test Results}

A large set of numerical experiments has been performed on this test model, using all four modal corrections mentioned in Step 8, as well as some others. We present illustrative and representative results here. It should be mentioned that, although our test model includes various controllers, it does not include protective relaying, so the transients seen here may not reflect what might be seen in practice with such a system. However, the lack of protective relaying should generally make the challenge to SME in our tests all the more severe, so we do not see this as a serious limitation on the tests.

Figure 4 shows an example of a frequency-domain test (in MATLAB) that compares the Bode magnitude responses of the linearized unreduced and reduced models. The input is the mechanical power perturbation at generator 17 , and the output is the perturbation in the absolute (i.e. not relative) angle at this machine. It is evident that even the no-correction SME equivalent matches the response of the unreduced model over much of the frequency range. Adding in a correction for just the zero mode causes the remaining discrepancy to largely disappear. The external-basis/full-mode equivalent (not shown here) and full-basis/full-mode equivalent perform comparably with each other, and are very good. If all angles (in both the unreduced and reduced models) are not measured as absolute angles, but are measured relative to some reference machine in the study group, then even the no-correction 

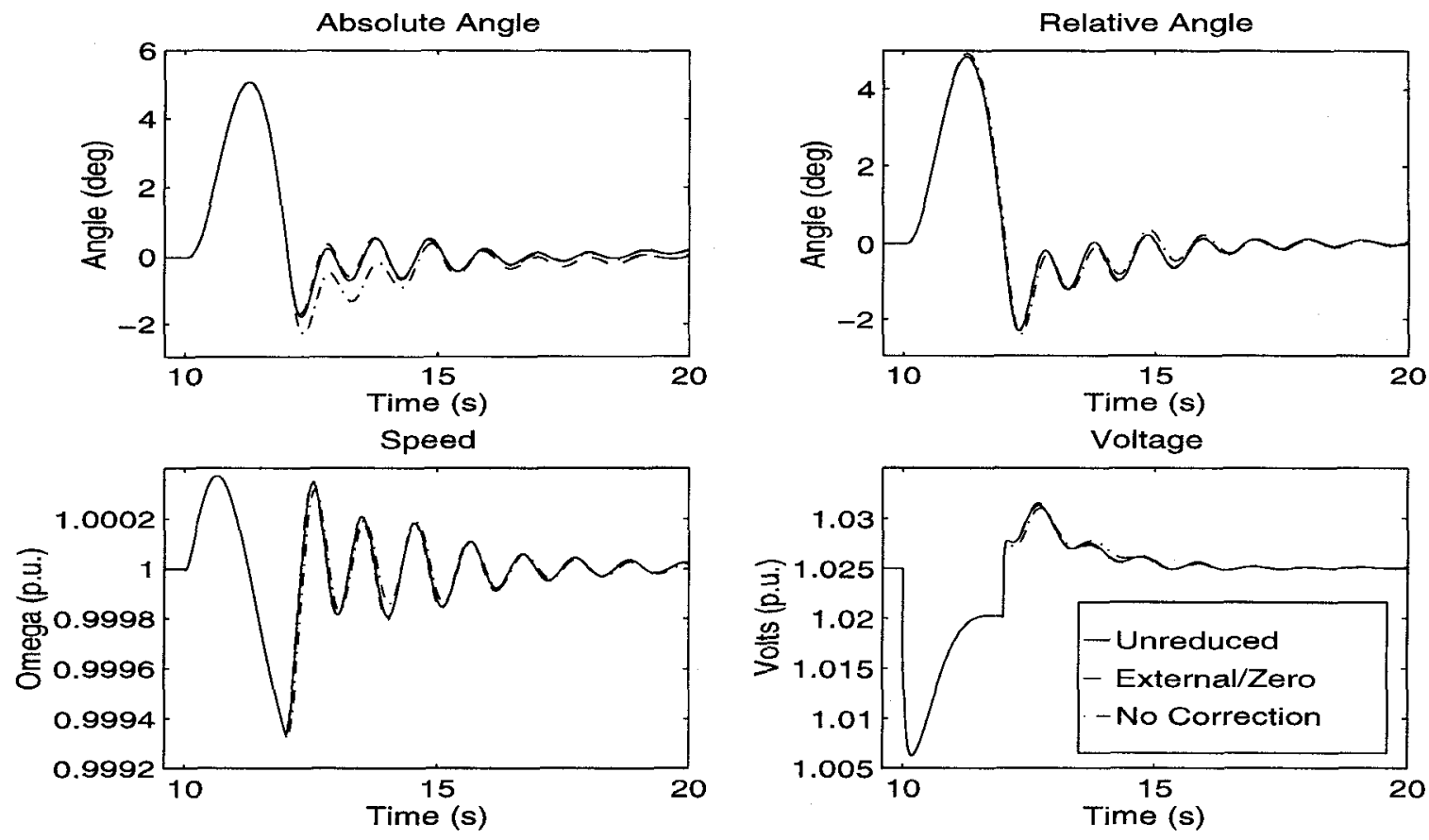

Figure 5: Perturbation on Generator 17. The perturbation is a disconnection of the generator during 2 seconds. For the unreduced model, the reduced model with the external-basis/zero-mode correction, and the no-correction reduced model, the plots show the absolute angle, the relative angle (referenced to the study-group basis generator) and the rotor speed of the perturbed generator, as well as the voltage at the generator terminal bus. (The angles are shown as deviations from nominal.)

equivalent turns out to perform very well. These general observations about the results apply to essentially all our experiments, so we shall not repeat them each time. We also performed time-domain tests (not shown here) on the linearized models; simulation times for the reduced models were significantly smaller - by factors in the range of approximately 6 to 12 - than the times for the unreduced model.

We turn now to time-domain simulations of the nonlinear models, all carried out using the variable-time-step routines of EUROSTAG. Figure 5 shows the results obtained when generator 17 is totally disconnected between $t=10 \mathrm{~s}$ and $t=12 \mathrm{~s}$. We present only the no-correction and external-basis/zero-mode cases, but the other reduced models perform comparably. The no-correction case turns out to be only slightly worse than the others, which emphasizes again the importance of an efficient partitioning, and shows that synchrony-based decomposition of the system has produced very well defined groups. The modal correction allows the reduced model to match the unreduced system more closely, producing a response that is virtually indistinguishable from that of the unreduced model over the full 10-second window. Simulation times for the reduced model were around $27 \%$ smaller than those for the unreduced model. More significant reductions in simulation times may be expected for larger systems, where a bigger fraction of the machines on the system will probably end up being replaced.

The results of another time-domain experiment are presented in Fig. 6. The perturbation here is a permanent short circuit in the middle of the line between generators 17 and 22 , which corresponds in effect to a topological change in the network. The reduced models with the external-basis/zero- mode correction and with no correction are compared to the unreduced model. The plots show the the absolute angles, the relative angles (referenced to machine 21), and the terminal bus voltages of the two generators. Good performance is seen throughout. Simulation times for the reduced model were around $19 \%$ less than for the unreduced model.

We have shown how absolute angles, and not just relative angles, are rather well preserved by SME. For most purposes, it would suffice to preserve the relative angles. However, if one wished for some reason (e.g. protection) to compare actual angle measurements made outside the study group with the predictions of a reduced model for the study group, it would be important to preserve absolute angles.

\section{Conclusion}

We have presented an overall perspective on SME, including some recent extensions and simplifications, as well as illustrative tests on an intermediate-sized model (larger and more detailed than in earlier tests of SME). The SME equivalent is obtained cleanly and directly, and the results are promising. In particular, the good performance of the zero-mode correction opens the door to carrying out SME on much larger models, without the burden of computing modes for these large models.

Some important issues remain to be addressed, even at the level of intermediate-sized models. Note that our synchronic grouping tells us how to group the generators, and the theoretical development of the SME equivalent gives some assurance that the equivalent will perform well for perturbations on the study-group generators. For perturbations at load buses and 
Abs. Angle - Gen. 22

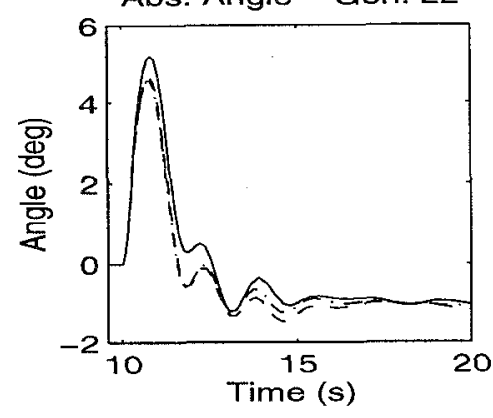

Abs. Angle - Gen. 17

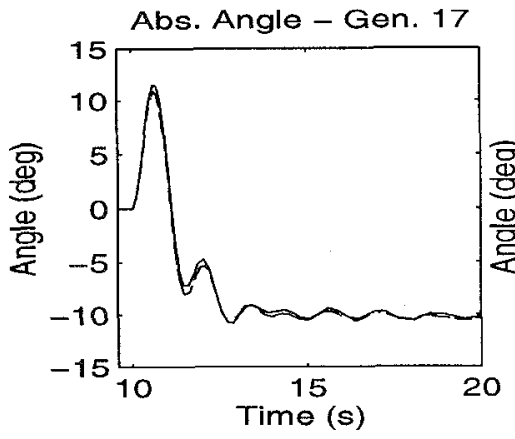

Rel. Angle - Gen. 22

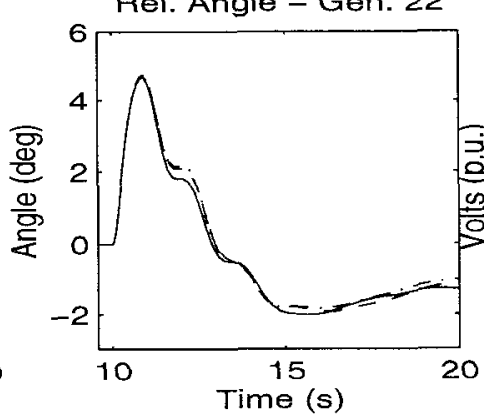

Rel. Angle - Gen. 17

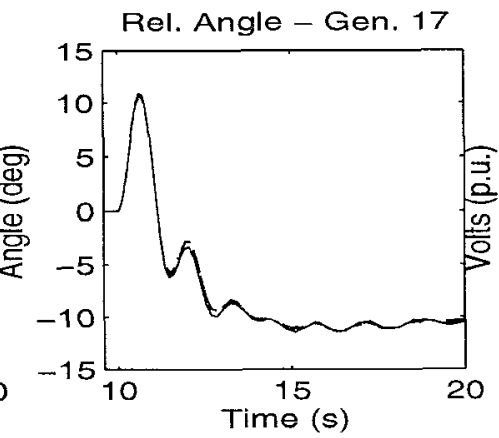

Voltage - Gen. 22

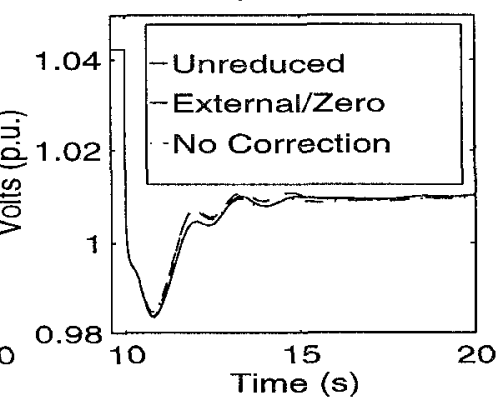

Voltage - Gen. 17

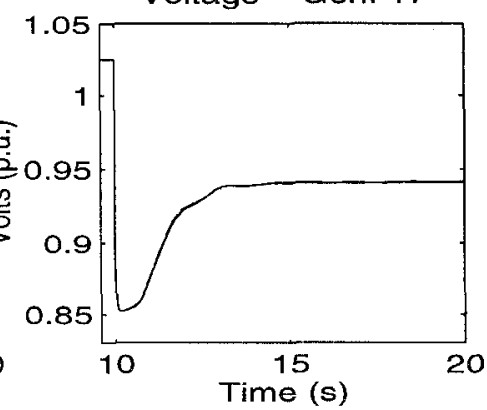

Figure 6: Permanent Short Circuit On Line Connecting Generators 17 and 22. The plots show the absolute angles, the relative angles (referenced to the study-group basis machine), and the terminal bus voltages of generators 22 and 17 , for the unreduced model, the external-basis/zero-mode reduced model, and the no-correction reduced model. (All angles are shown as deviation from nominal.)

transmission lines that are clearly embedded within a particular study group, e.g. a line connecting two generators in the study group, we expect again to get good results. All the perturbations presented in this paper have been of the preceding types. Consider, however, a perturbation on a heavily loaded line between generators in two different synchronic groups, only one of which has been selected for the study group. We have simulations that show the possibility in this case of very poor matches between the responses of the full model and the SME equivalent. Clearly, what is needed in this situation is to expand the definition of the study group to include both the synchronic groups rather than just one of them, as noted in Step 5. In other situations, the solution may be less clear. Further research is needed to show how to factor into the definition of a study group whatever information is available about which buses and lines are to be perturbed or faulted in a given study. Related to this is the question of how to associate load buses and transmission lines with synchronic groups. In the context of slow-coherency, references [18] and [19] represent two approaches to addressing this task. From the point of view of synchrony, the discussion in Step 5 points to the considerations that are involved, and we expect to investigate these issues further.

\section{ACKNOWLEDGMENTS}

This paper has grown out of a project at the Laboratory for Electromagnetic and Electronic Systems, MIT, funded by Electricité de France (EDF). The authors are grateful to Dr. Bruno Meyer of EDF for his initiation, support and guidance of the project.

\section{REFERENCES}

[1] Kundur, P. et al. (Ontario Hydro), Dynamic Reduction, Version 1.0, Vol. 1: Final Report on EPRI Project 2447-01, April 1993.

[2] Germond, A. J and Ponmore, R., "Dynamic Aggregation of Generating Unit Models," IEEE Trans. Power Apparatus and Systems, Vol. PAS-97, No. 4, pp. 1060-1069, July/August 1978.

[3] CHow, J. H., Time-Scale Modeling of Dynamic Networks with Applications to Power Systems, Lecture Notes in Control and Information Sciences, Vol. 46, Springer Verlag, 1982.

[4] Zaborsky, J., Whang, K.-W., Huang, G.M., Chiang, L.-J., and LIN, S.-Y., "A Clustered Dynamical Model for a Class of Linear Autonomous Systems Using Simple Enumerative Sorting," IEEE Trans. Circuits and Systems, Vol. CAS-29, No. 11, pp. 747-757, November 1982.

[5] CHow, J.H., "New Algorithms for Slow Coherency Aggregation of Large Power Systems," in Systems and Control Theory for Power Systems, IMA Volumes in Mathematics and its Applications, Springer-Verlag, Vol. 64, pp. 95-115, 1995.

[6] Chow, J. H., Galrza, R., Accari, P. and Price, W. W., "Inertial and Slow Coherency Aggregation Algorithms for Power System Dynamic Model Reduction," IEEE Trans. Power Systems, vol 10, pp. 680-685, 1995.

[7] De Oliveira, S. E. M., De Queiroz, J. F., and Massaud, A. G. "Modal Dynamic Equivalent for Electric Power Systems: Parts I and II," IEEE Trans. Power Systems, Vol. 3, No. 4, pp.1723-1737, November 1988.

[8] Ramaswamy, G. N., Verghese, G. C., Rouco, L., LesievTRE, B, C., and FutĥATRE, O., "Synchronic Modal Equivalencing (SME): A New Framework for Constructing Dynamic Equivalents in Power Systems," North American Power Symposium, Manhattan, Kansas, September 1994. 
[9] Ramaswamy, G. N., Rouco, L., Fillâtre, O., Verghese, G. C., Panclatici, P., Lesieutre, B. C., and Peltier, D., "Synchronic Modal Equivalencing (SME) for Structure-Preserving Dynamic Equivalents," IEEE Trans. Power Systems, Vol. 11, pp.1929, February 1996.

[10] Ramaswamy, G. N., Modal Structures and Model Reduction, with Application to Power System Equivalencing, PhD thesis, EECS Department, Massachusetts Institute of Technology, June 1995.

[11] Ramaswamy, G. N., Verghese, G. C., and Lesieutre, B. C., "Multi-Dimensional Synchrony and Dynamic Equivalencing," 4th IEEE Conference on Control Applications, Albany, NY, pp. 605610, September 1995.

[12] Ramaswamy, G. N., Verghese, G. C., Rouco, L., Vialas, C., and DeMarco, C. L., "Synchrony, Aggregation, and Multi-Area Eigenanalysis," IEEE Trans. Power Systems, Vol. 10, pp. 19861993, November 1995.

[13] Pérez-Arriaga, I. J., Verghese, G. C., Pagola, F. L., Sancha, J. L. and SchwepPE, F. C., "Developments in Selective Modal Analysis of Small-Signal Stability in Electric Power Systems," Automatica, Vol. 26, No 2, pp. 215-231, 1990.

[14] Castrillón-Candás, J. E., Optimal Reduced Order Modeling of Power Systems Based on Synchronic Modal Equivalencing, S.M. thesis, EECS Department, Massachusetts Institute of Technology, February 1996.

[15] Vernotte, J.F., Panciatici, P., Antoine, J.P., Deuse, J., and STUBbe, M., "High Fidelity Simulation of Power System Dynamics," IEEE Computer Applications in Power, January 1995.

[16] Astic, J. Y., Bihain, A., and Jerosolimski, M., , "The Mixed Adams - BDF Variable Step Size Algorithm to Simulate Transient and Long Term Phenomena in Power Systems," IEEE Transactions on Power Systems, Vol. 9, No. 2, pp. 929-935, May 1994.

[17] The Math Works, INC., Matlab Users's Guide, Natick, MA, 1994.

[18] Yusof, S. B., Rogers, G. J., and Alden, R. T. H., "Slow Coherency Based Network Partitioning Including Load Buses," IEEE Trans. Power Systems, Vol. 8, pp. 1375-1382, August 1993.

[19] Demarco, C. L., and Wasner, J., "A Generalized Eigenvalue Perturbation Approach to Coherency," 4th IEEE Conference on Control Applications, Albany, NY, pp. 611-617, September 1995.

\section{BIOGRAPHIES}

Ganesh N. Ramaswamy (S'90, M'95) was born in Kuala Lumpur, Malaysia. He received his Bachelor's and Master's degrees, his Engineer's degree and his Ph.D., all in Electrical Engineering and all from MIT, in 1992, 1993 and 1995, respectively. He joined the IBM Thomas J. Watson Research Center in 1995 as a Research Staff Member. His research interests include modeling and identification of large dynamic systems, pattern recognition and clustering, statistical signal processing, and communication systems. He is a member of Tau Beta Pi, Eta Kappa $\mathrm{Nu}$, and Sigma Xi.

Christophe Evrard received his engineering degree from the Ecole des Mines de Paris in 1994. He was at MIT as a visiting engineer from Electricité de France (EDF) from November 1994 till February 1996, and is presently back at EDF.

George C. Verghese (S '74, M '78) received his B.Tech. from IIT, Madras, M.S. from SUNY, Stony Brook, and Ph.D. from Stanford University. He joined the EECS Department at MIT in 1979, where he is Professor of Electrical Engineering, attached to the Laboratory for Electromagnetic and Electronic Systems. He has served as Associate Editor of Automatica and of the IEEE Trans. Automatic Control, and is currently an Associate Editor for the IEEE Trans. Control Systems Technology. He has also served the IEEE Power Electronics Society in various capacities, including as a member of its AdCom. He is coauthor (with J.G. Kassakian and M.F. Schlecht) of Principles of Power Electronics, Addison-Wesley, 1991.

Ollivier Fillâtre ( $M$ ' 95 ) received his engineering and doctoral degrees from the Ecole Centrale de Paris in 1988 and 1991 respectively, with a specialization in numerical analysis from Paris VI University in 1988. He has been with EDF since 1992, working with EDF's R\&D Division on power system simulation where he is presently the EUROSTAG team leader.

Bernard C. Lesieutre (S ' $86, \mathrm{M}$ '93) received his B.S., M.S., and $\mathrm{Ph} . \mathrm{D}$. degrees in electrical engineering from the University of Illinois at Urbana-Champaign in 1986, 1988, and 1993 respectively. He is currently an Assistant Professor of Electrical Engineering at the Massachusetts Institute of Technology. His research interests include machine modeling. and power system dynamics, stability and control. He is a member of Eta Kappa Nu. 